\title{
Limiting performance analysis of underwater shock isolation of a system with biodynamic response using genetic algorithm
}

\author{
Z. Zong ${ }^{\mathrm{a}}$, K.Y. Lam ${ }^{\mathrm{a}}$ and Tessa Gan ${ }^{\mathrm{b}}$ \\ ${ }^{\mathrm{a}}$ Institute of High Performance Computing, 89C, \\ Science Park Drive 02-11/12, The Rutherford, \\ Singapore Science Park I, Singapore 118261, \\ Singapore \\ Tel.: +65 776167; Fax: +65 7761962; E-mail: \\ zongzhi@ihpc.nus.edu.sg; lamky@ihpc.nus.edu.sg \\ ${ }^{\mathrm{b}}$ Naval Logistics Department, MINDEF Building, \\ Gombak Drive, Singapore 669645, Singapore \\ E-mail: tlkkgan@singnet.com.sg
}

Received 24 February 2000

Revised 26 June 2000

Biodynamic response of shipboard crew to underwater shock is of a major concern to navies. An underwater shock can produce very high accelerations, resulting in severe human injuries aboard a battleship. Protection of human bodies from underwater shock is implemented by installing onboard isolators. In this paper, the optimal underwater shock isolation to protect human bodies is studied. A simple shock-structure-isolator-human interaction model is first constructed. The model incorporates the effect of fluid-structure interaction, biodynamic response of human body, isolator influence. Based on this model, the optimum shock isolation is then formulated. The performance index and restriction are defined. Thirdly, GA (genetic algorithm) is employed to solve the formulated optimization problem. GA is a powerful evolutionary optimization scheme suitable for large-scale and multi-variable optimization problems that are otherwise hard to be solved by conventional methods. A brief introduction to GA is given in the paper. Finally, the method is applied to an example problem and the limiting performance characteristic is obtained.

Keywords: Biodynamic response, underwater shock, optimal isolation, GA (genetic algorithm)

\section{Introduction}

An underwater shock produces two important effects. The first effect is to destroy a battleship so that she cannot continue her functions. The second is to injure onboard personnel through dynamic structural responses. Underwater shock and its effects on marine structures has been an active research field since World War II. Commercial codes are available for predicting the structural responses to a nearby or far away charge [4,9], and some numerical attempts were made to simulate structural responses to underwater shock $[12,14,15]$. Comparatively speaking, we know much less about the effect of underwater shock on the human body. Recently, there is a growing interest in the effect of underwater shock on onboard human bodies due to the fact that crew survivability is of a major concern to all navies $[7,8,10]$. These studies lead to the following conclusions:

1. An underwater shock can produce very high accelerations, which are far beyond the tolerance limits of the human body; and

2. There exists the possibility that the ship survives underwater shock, but the onboard personnel are severely injured. This is because the human body can withstand much smaller accelerations than some structures do.

Therefore, a new factor is introduced in ship design against underwater shock, that is, optimum isolation design for protecting human bodies from underwater shock.

Underwater shock isolators act to reduce the hazardous effects of shock disturbances on human bodies via structural motions. The problem of optimum design is defined as the selection of isolators that cause the performance index of the system to be optimized. Optimum design is considered at two levels: at a lower level, a pre-selected class of isolators is defined, and the 
optimum isolator is found from this pre-selected class. The sense of optimization in this case is totally dependent on the choice of the class of candidate isolators, and some isolator not in this class may well be better than the optimum. The optimization at this level is device-oriented and directed to design. Thus, this problem is termed design-parameter synthesis. At a higher optimization level, the lower bound value is found for any type of isolator. The resulting value of the performance index is the best that can be accomplished for any isolator regardless of the hardware device utilized. Thus, this process is called limiting performance synthesis. The difference between this limiting optimum and the former design parameter optimum represents the margin for improvement in system performance, which theoretically can be achieved with an isolator concept [11].

The most advanced achievements in the theory of optimum impact and shock isolation have been made for a single-degree-of-freedom model, and a comprehensive presentation of the state-of-the-art of the theory of optimal shock isolation is given in [1]. Although these studies are restricted to shock and impact isolation of a mechanical system, the theory is undoubtedly applicable to a system with human response to underwater shock. To apply the theory to the optimal limiting performance synthesis of underwater shock isolation of a system with biodynamic response, two fundamental problems should be addressed. The first problem is the modeling of shock-structure-isolator-human interaction, and the second is the solution of the problem.

In this paper, the limiting performance synthesis of a system with biodynamic response subjected to underwater shock is studied using GA. First, a simple shock-structure-isolator-human interaction model is constructed. The model incorporates the effect of fluid-structure interaction, biodynamic response of human body and isolator influence. Based on this model, the optimum shock isolation problem is then formulated. The performance index and restriction are defined. Thirdly, GA (genetic algorithm) is used to solve the formulated optimization problem. GA is a powerful evolutionary optimization scheme suitable for largescale and multi-variable optimization problems, which are otherwise hard to be solved by conventional methods. A brief introduction to GA is given in the paper. Finally, the limiting performance characteristic of such a system is obtained using the proposed method.

\section{The shock-structure-isolator-human interaction model and the optimal shock isolation synthesis}

Consider a ship structure with onboard personnel. The structure is in contact with water on one side, and is equipped with an isolation device on the other side. On the isolator is a human body of weight $m_{H}$. Suppose an underwater shock produced by a charge of weight $G$ at standoff distance $R$ strikes the structure from water. The shock deforms the structure, which in turn transmits the shock disturbance to the human body via the isolator. The purpose here is to find the optimum isolation design for such a system.

This is a complicated interaction system, which involves shock-structure interaction, shock absorption by isolator, isolator-human interaction. Solution of such a problem requires high-speed computers. To simplify the problem as much as possible without losing essential features, consider a single-degree-of-freedom (SDF) system as shown in Fig. 1. Instead of analyzing a real 3-D ship, we use an infinite plate to represent the structure. The mass of the plate is $m_{P}$. Suppose the charge is sufficiently away from the plate so that the shock wave near the plate is in fact planar. The spring-dashpot model shown in Fig. 1(a) is the simplest isolator. To be general, we use force function $u(t)$ to represent an isolator as shown in Fig. 1(b). The human body is a very complicated biological and mechanical system, modeling of which is still under intense studies. From the viewpoint of shock isolation, however, the simplest model of the human body is a rigid mass $m_{H}$. In [2] is discussed the condition for this model of human body to be applicable. We'll establish the governing equations based on the model shown in Fig. 1(b).

\subsection{Isolation system}

Using the model shown in Fig. 1(b), we assume that the net force across the isolator is an unknown function of time $u(t)$. The equation of motion for the human body is

$$
m_{H} \ddot{z}+u(t)=0
$$

with the kinetic condition

$$
z(t)=x(t)+y(t)
$$

in the interval of interest $0 \leqslant t \leqslant t_{f}$. Here, $z(t)$ is the displacement of the human body, $x(t)$ is the rattlespace (i.e., the maximum displacement of the isolator relative to the plate) of the isolator and $y(t)$ is the displacement of the plate. The initial conditions are specified as

$$
z(0)=\dot{z}(0)=0
$$




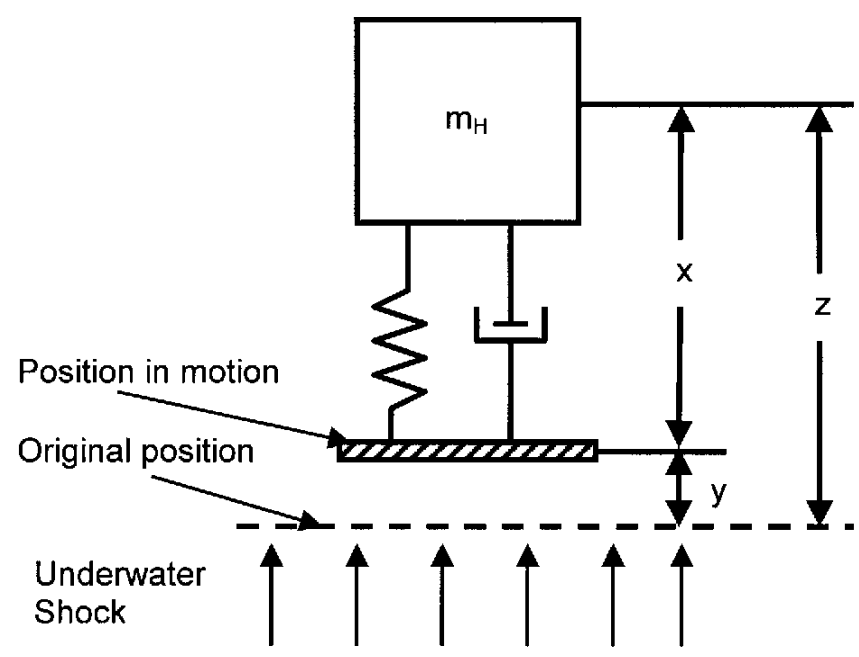

(a) A SDF system modeling shock-structure-isolator-human interaction

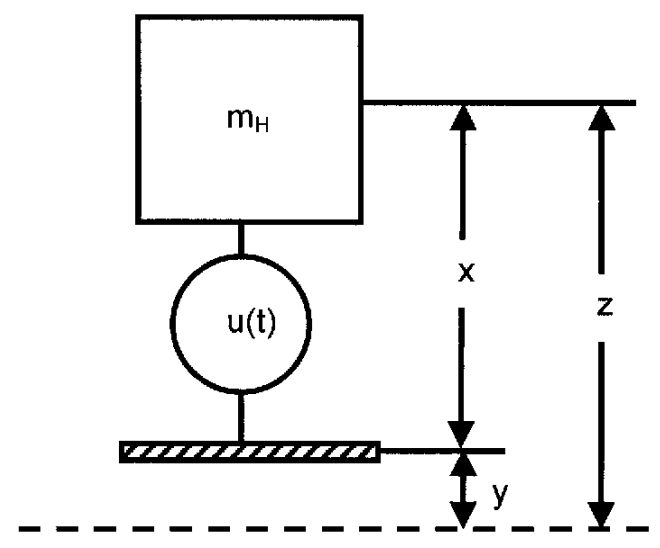

(b) The corresponding generic SDF system

Fig. 1. The simplified SDF system modeling the shock-structure-isolator-human interaction.

\subsection{Shock-structure interaction}

An underwater explosion shock wave is characterized by a sharp rise in pressure followed by a quick decay in time. The pressure-time curve can be approximated by an exponential function of the form [3]

$$
P_{I}=P_{m} e^{-t / \tau}
$$

where the similarity solutions for peak pressure $P_{m}$ and time decay $\tau$ are

$$
\begin{aligned}
& P_{m}=2.786 \times 10^{7}\left(\frac{G^{1 / 3}}{R}\right)^{1.18}\left(\mathrm{~N} / \mathrm{m}^{2}\right), \\
& \tau=0.5818 G^{1 / 3}\left(\frac{G^{1 / 3}}{R}\right)^{-0.185} \quad \text { (microsecond). }
\end{aligned}
$$

Here, $G$ is charge weight in $\mathrm{kg}$ and $R$ is standoff distance in meter.

On arrival at the plate, the shock wave produced by the charge (see Eq. (4)) strikes the plate, giving it an initial velocity. A reflected wave of pressure will at the same time be transmitted back into the water, which must be of such magnitude that the resultant particle velocity of the water in contact with the plate is equal to the velocity of the plate. Assume $P_{R}$ is the pressure in the reflected wave, the equation of motion of the plate is

$$
m_{P} \ddot{y}-u(t)=\left(P_{I}+P_{R}\right) S,
$$

where $P_{I}$ is given by Eq. (4), and $S$ is the plate area. Linear superposition principle allows us to decompose $y$ into the sum of two components: 


$$
y=y_{0}+Y,
$$

where $y_{0}$ is the displacement in the absence of the isolation system and the human body, satisfying

$$
m_{P} \ddot{y}_{0}=\left(P_{I}+P_{R}\right) S,
$$

and the boundary conditions:

$$
\dot{y}_{0}=V_{I}+V_{R}, \quad(y=0) .
$$

Here $V_{I}$ and $V_{R}$ are the particle velocities in the incident and reflected waves. For plane waves, $V_{I}=$ $P_{I} / \rho c, V_{R}=-P_{R} / \rho c$, where $\rho$ and $c$ are water density and the sound speed in water, and hence we have

$$
\begin{array}{ll}
\dot{y}_{0}=\frac{P_{I}-P_{R}}{\rho c}, & (y=0) \\
\dot{y}_{0}=0, & (y=0, t=0) .
\end{array}
$$

Solving Eqs. (8) and (10) using standard methods, we obtain

$$
\begin{aligned}
y_{0}= & \frac{2 P_{m} \tau S}{m_{P}(1-\beta)}[\exp (-\beta t / \tau) \\
& -\exp (-t / \tau)],(t>0) \\
P_{R}= & \frac{P_{m}}{1-\beta}[(1+\beta) \exp (-\beta t / \tau) \\
& -2 \beta \exp (-t / \tau)],(t>0)
\end{aligned}
$$

where $\beta=\rho c \tau S / m_{P}$.

$Y(t)$ in Eq. (7) is the displacement due to force function $u(t)$, satisfying

$$
m_{P} \ddot{Y}=u(t)
$$

Once $u(t)$ is given, $Y(t)$ can be integrated from the equation above. Equations (1)-(3), (7), (11) and (12) define the SDF shock-structure-isolator-human interaction model.

\subsection{Tolerance criteria for whole body}

A series of experiments $[5,13]$ led to the link between human tolerance and acceleration. Those data provide useful early guidelines for safety assessments of crew survivability in the event of an underwater explosion. More recent tests have led to other acceleration formulas for regional injury tolerances, such as head injury tolerance, chest injury tolerance. In Fig. 2 is summarized the relationship between human whole-body tolerance and acceleration based on the experimental data in [5] for two acceleration directions: spine-ward and head-ward. The duration in the event of underwater shock is extremely short, of the order of 1-100 ms. Within this range, the tolerance limits are almost con- stant. Therefore, on assessing human tolerance to underwater shock, peak acceleration is the most important factor to be considered. From Fig. 2(a) for spine-ward acceleration, human body is safe if peak acceleration is below $50 \mathrm{~g}$, where $g$ is the gravity acceleration. Above $50 \mathrm{~g}$, human body is prone to suffer from moderate to severe injuries. From Fig. 2(b) for head-ward acceleration, human body is safe if the peak acceleration is below $18 \mathrm{~g}$. Therefore, the tolerance is case-dependent. Using $A$ to denote the acceleration tolerance, we know that the peak acceleration of human body must satisfy

$$
\max |\ddot{z}| \leqslant A \text {. }
$$

From Eq. (2) we have

$$
\begin{aligned}
& \max \left|\frac{u(t)}{m_{H}}\right| \leqslant A, \text { or, } \\
& -m_{H} A \leqslant u(t) \leqslant m_{H} A .
\end{aligned}
$$

This is the constraint on the force function $u(t)$.

\subsection{Optimal shock isolation system}

The rattlespace $x(t)$ refers to the maximum displacement of the isolation system plus the human body relative to the plate. It is an important performance index in isolation design. In comparing isolators with different performances, only those that satisfy the acceleration bound Eq. (14) would be considered acceptable designs, and among those the one requiring the least rattlespace would be the optimum. Writing it in mathematical form, we have

Find force function $u(t)$ such that

$$
\begin{aligned}
& \max |x(t)| \rightarrow \min \\
& \text { subject to: }-m_{H} A \leqslant u(t) \leqslant m_{H} A,
\end{aligned}
$$

where,

$$
\begin{aligned}
& x(t)=z(t)-y_{0}(t)-Y(t), \\
& m_{H} \ddot{z}=-u(t), \\
& m_{P} \ddot{Y}=u(t) .
\end{aligned}
$$

\section{Influence of the force function on the rattlespace}

In this part, a simple example is given to show the influence of the force function $u(t)$ on the rattlespace $x(t)$. The example exhibits how the different time histories of the force affect the history of rattlespace. The example considered is as follows: 


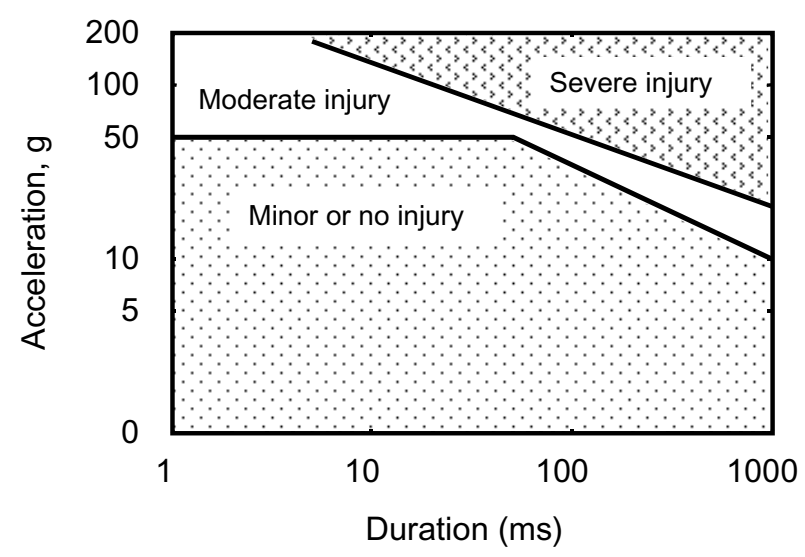

(a) Tolerance to spineward acceleration

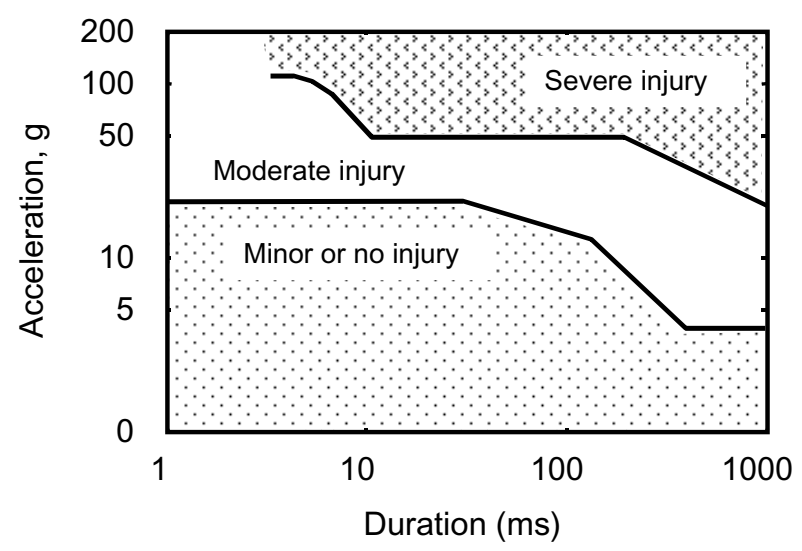

(b) Tolerance to headward acceleration

Fig. 2. Whole-body tolerance criterion to acceleration [13].

$$
\begin{aligned}
& \ddot{z}+u=0, \\
& z=x+y, \\
& y=0.01 \exp (-t / \tau), \\
& \tau=4.88 \times 10^{-3}, \\
& |\dot{z}| \leqslant 100,
\end{aligned}
$$

where $u(t)$ is a step function defined by

$$
u=\left\{\begin{array}{l}
u_{1}, 0 \leqslant t \leqslant \frac{0.01}{3} \\
u_{2}, \frac{0.01}{3} \leqslant t \leqslant \frac{0.02}{3} \\
u_{3}, \frac{0.02}{3} \leqslant t \leqslant 0.01
\end{array}\right.
$$

In concise form, $u=\left(u_{1}, u_{2}, u_{3}\right)^{T}$, where $T$ denotes transpose of the vector. From Eq. (16), we know $-100 \leqslant u_{i} \leqslant 100,(i=1,2,3)$.
If we take $u=(100,100,100)^{T}$, the time history of the rattlespace can be obtained by integrating Eq. (16). The result is shown in Fig. 3 using the dashed line. It is a monotonically increasing function with time. Similarly, we obtain the time histories of the rattlespace for $u=(-100,-100,-100)^{T}$ and $u=(10,20,30)^{T}$. The results are shown in Fig. 3 using the dashed-dotted line and solid line, respectively. Both curves are nonmonotonically curves with time. For different force functions, the rattlepsace responses are different.

From the figure, we have following relations for $0 \leqslant$ $t \leqslant 0.01(\mathrm{~s})$ :

$$
\begin{aligned}
\max |x(t)|= & 10, \text { for } u=(100,100,100)^{T}, \\
\max |x(t)|= & 12.5 \\
& \text { for } u=(-100,-100,-100)^{T}, \\
\max |x(t)|= & 10, \text { for } u=(10,20,30)^{T} .
\end{aligned}
$$




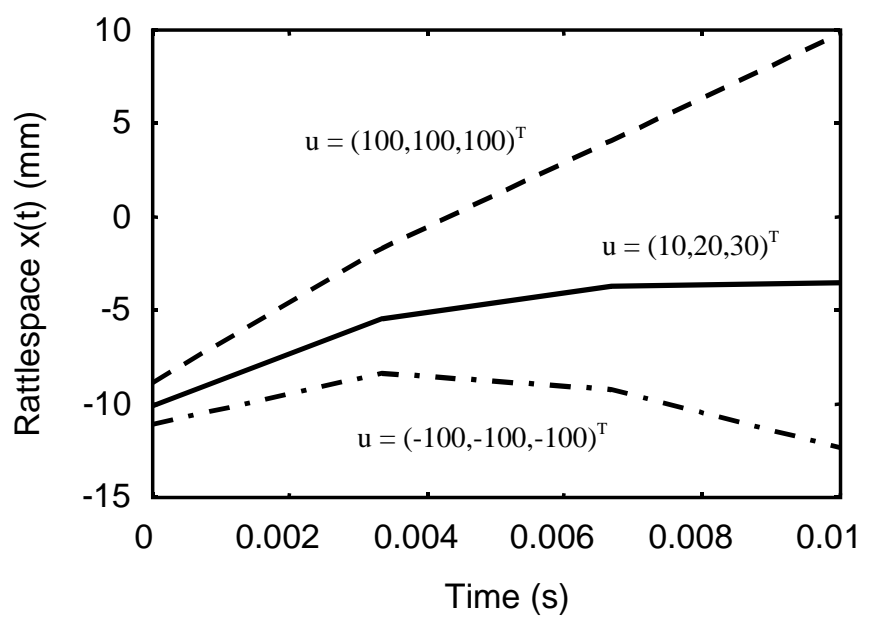

Fig. 3. Influence of $u(t)$ on the rattlespace $x(t)$.

Thus, the first and the third force functions both give the least rattlepsace.

\section{Optimum synthesis of underwater shock isolation using GA}

\subsection{A brief introduction to $G A$}

In [11], several methods are introduced to solve Eq. (15). In this paper, GA (genetic algorithm) is used to solve Eq. (15) because GA is so powerful that it is able to solve many difficult-to-solve optimization problems by conventional optimization techniques. Thus, a brief introduction is first given to GA. GA is a stochastic search technique imitating the mechanism of natural selection and natural genetics. Instead of starting from one initial value, GA starts from a set of random initial values, which are called population. Each value in this population that is a possible solution, is a chromosome. A chromosome, usually written in the binary form, is a string of symbols ( 0 and 1 in binary form). The chromosomes evolve through successive iterations, called generations. During each generation, the chromosomes are evaluated using the measure of fitness, which is in fact the objective function value $\max |x(t)|$ in Section 2 . To create next generation, new chromosomes, called offspring are formed by either (a) merging two chromosomes from current generation using a crossover operator, or (b) modifying a chromosome using a mutation operator. Thus, a new generation is formed by selecting some of the parents and offspring with fitter values, and rejecting others so as to keep the population size constant (see Fig. 4).
Fitter chromosomes have higher probabilities to be selected. After several generations, the algorithms converge to the best chromosome, which hopefully, represents the optimum solution to the problem.

From Fig. 4, there are two kinds of operations in GA

1. Genetic operation: crossover and mutation

2. Evolution operations: selection.

Crossover is the main operator, which operates on two chromosomes at a time and generates offspring by combining both chromosomes' features. A simple way to achieve crossover is to choose a random cut-point and generate the offspring by combining the segment of one parent to the left of the cut-point with the segment of the other parent to the right of the cut-point. This method works well with the bit string representation. The performance of GA depends largely on the performance of the crossover operator used.

The crossover rate is defined as the ratio of the number of offspring produced in each generation to the population size. A higher crossover rate allows exploration of more of the solution space, but may waste a lot of computation time on exploring unpromising regions of the solution space.

Mutation produces spontaneous random changes in various chromosomes. A simple way to achieve mutation is to change one or more genes, which serves replacing the genes lost from the population during the selection so that they can be tried in a new context or providing the genes that were not present in the initial population.

The mutation rate is defined as the percentage of total number of genes in the population, which controls the rate at which new genes are introduced into the 


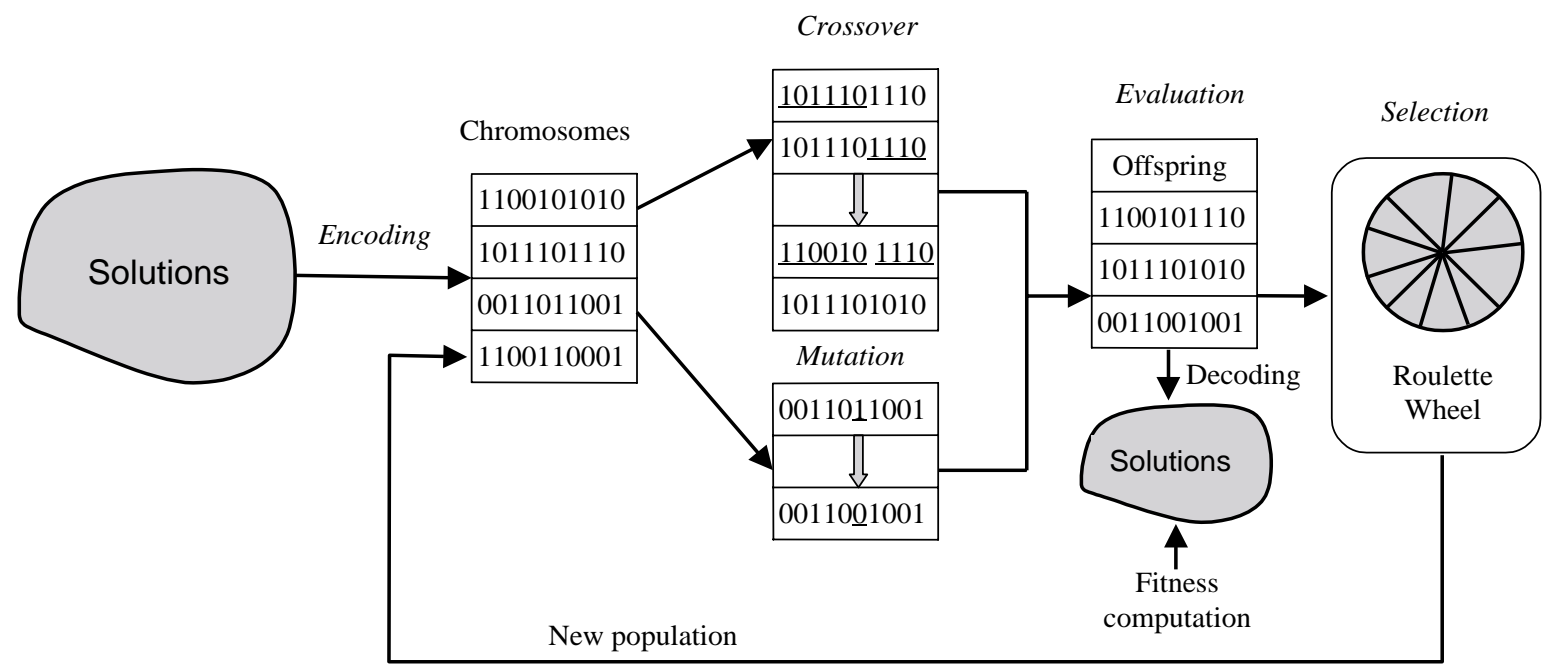

Fig. 4. The general structure of genetic algorithm.

population for trial. Higher mutation rate introduces too much random perturbation and lower mutation rate may reject many genes that are never tried out.

In general, GA is different from conventional optimization and search procedures. The main advantages are

1. GA does not require derivatives and apply to any kind of objective functions with any constraints;

2. The ergodicity of evolution operations makes GA very effective at performing global search [6].

\subsection{An example: Minimization of Ackley's function}

Ackley's function is a continuous and multimodal test function obtained by modulating an exponential function with a cosine wave of moderate amplitude [6]. Its topology, as shown in Fig. 5, is characterized by an almost flat outer region and a central hole or peak where modulations by the cosine wave become more and more influential. Ackley's function is defined as follows:

$$
\begin{aligned}
f\left(x_{1}, x_{2}\right)= & -c_{1} \exp \left[-c_{2} \sqrt{\frac{1}{2} \sum_{i=1}^{2} x_{i}^{2}}\right] \\
& -\exp \left[\frac{1}{2} \sum_{i=1}^{2} \cos \left(c_{3} x_{i}\right)\right]+c_{1}+e, \\
& \left|x_{i}\right| \leqslant 5, i=1,2,
\end{aligned}
$$

where $c_{1}=20, c_{2}=0.2, c_{3}=2 \pi$, and $e=2.71282$. This function has many local optimums, but has one global optimum. The known global optimal solution is $\left(x_{1}^{*}, x_{2}^{*}\right)=(0,0), f\left(x_{1}^{*}, x_{2}^{*}\right)=0$. A search scheme, like hill-climbing, would surely get trapped in a local optimum. Thus this function provides a reasonable test case for GA.

The initial population is randomly created within the region $[-5,5]$ as follows:

$$
\begin{array}{lr}
x_{1} & \multicolumn{1}{c}{x_{2}} \\
\nu_{1}=\left(\begin{array}{lr}
0.61846, & -3.58974
\end{array}\right) \\
\nu_{2}=\left(\begin{array}{lr}
-4.01820, & 1.53218
\end{array}\right) \\
\nu_{3}=(2.91803, & 3.54335) \\
\nu_{4}=\left(\begin{array}{lr}
-1.7367, & 3.73592
\end{array}\right) \\
\nu_{5}=\left(\begin{array}{lr}
4.36552, & 7.78069
\end{array}\right) \\
\nu_{6}=\left(\begin{array}{lr}
-1.59600, & 4.01028
\end{array}\right) \\
\nu_{7}=\left(\begin{array}{lr}
1.79250, & 0.69811
\end{array}\right) \\
\nu_{8}=\left(\begin{array}{lr}
2.08029, & -1.30360
\end{array}\right) \\
\nu_{9}=\left(\begin{array}{ll}
-3.62606, & 2.31376
\end{array}\right) \\
\nu_{10}=(2.34367,
\end{array}
$$

The corresponding fitness function values are

$$
\begin{array}{ccc}
x_{1} & x_{2} & \begin{array}{c}
\text { Fitness } \\
\text { value }
\end{array}
\end{array}
$$

$\operatorname{eval}\left(\nu_{1}\right)=f(0.61846, \quad-3.58974)=20.8514$

$\operatorname{eval}\left(\nu_{2}\right)=f(-.4 .01820, \quad 1.53218)=21.2108$

$\operatorname{eval}\left(\nu_{3}\right)=f(2.91803, \quad 3.54335)=21.4625$

$\operatorname{eval}\left(\nu_{4}\right)=f(-1.7367, \quad 3.73592)=21.1242$

$\operatorname{eval}\left(\nu_{5}\right)=f(4.36552, \quad 7.78069)=21.5376$

$\operatorname{eval}\left(\nu_{6}\right)=f(-1.59600, \quad 4.01028)=21.1400$

$\operatorname{eval}\left(\nu_{7}\right)=f(1.79250, \quad 0.69811)=12.1992$

$$
\text { (min) }
$$

$\operatorname{eval}\left(\nu_{8}\right)=f(2.08029, \quad-.1 .30360)=15.4081$

$\operatorname{eval}\left(\nu_{9}\right)=f(-3.62606, \quad 2.31376)=21.6394$

$\operatorname{eval}\left(\nu_{10}\right)=f(2.34367, \quad-0.10086)=14.9258$ 


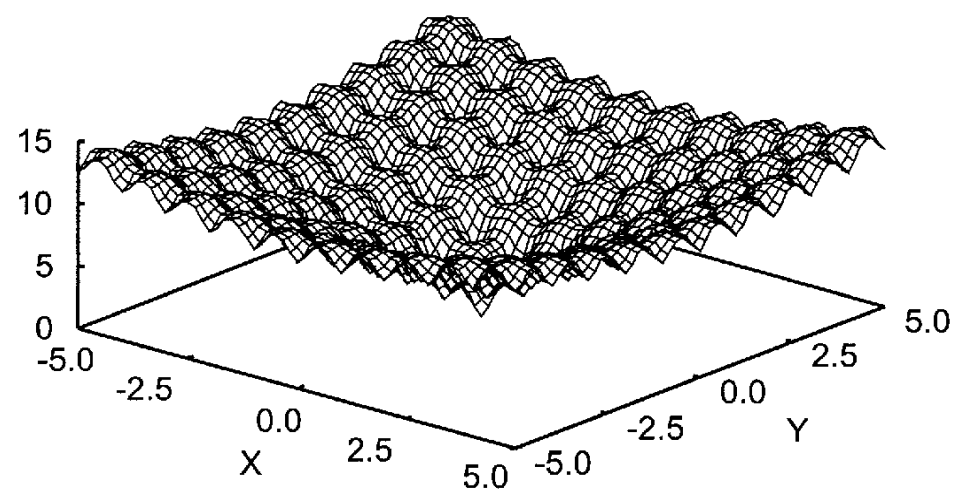

Fig. 5. Ackley's function.

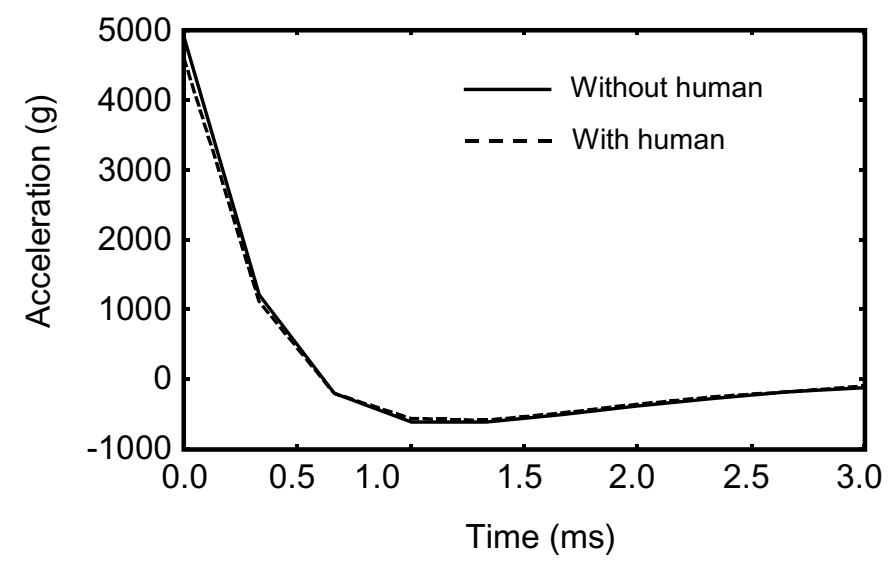

Fig. 6. Plate accelerations.

The optimal value is $f(1.79250,0.69811)=$ 12.1992. At the 200th generation, we have the following chromosomes:

$$
\begin{array}{ccc}
x_{1} & x_{2} \quad \begin{array}{c}
\text { fitness } \\
\text { value }
\end{array}
\end{array}
$$

$\operatorname{eval}\left(\nu_{1}\right)=f(0.03739, \quad-1.44398)=8.51925$

$\operatorname{eval}\left(\nu_{2}\right)=f(0.00015, \quad-0.00046)=0.00546$ (min)

$\operatorname{eval}\left(\nu_{3}\right)=f(1.70370, \quad-0.90655)=11.9033$

$\operatorname{eval}\left(\nu_{4}\right)=f(-1.69088, \quad 3.69686)=21.2710$

$\operatorname{eval}\left(\nu_{5}\right)=f(1.37715, \quad-0.95813)=10.1892$

$\operatorname{eval}\left(\nu_{6}\right)=f(-1.84805, \quad 3.45027)=20.9492$

$\operatorname{eval}\left(\nu_{7}\right)=f(-3.32789, \quad 1.19526)=20.1366$

$\operatorname{eval}\left(\nu_{8}\right)=f(1.04236, \quad 0.42894)=6.16863$

$\operatorname{eval}\left(\nu_{9}\right)=f(0.328532,-1.38478)=8.83215$

$\operatorname{eval}\left(\nu_{10}\right)=f(-0.94531, \quad 4.86755)=20.3244$

The optimal value is $f(0.00015,-0.00046)=$ 0.00546. At 1000th generation, the optimum is $f(0.000002,-0.0000)=-0.00546$.

\subsection{Optimum underwater shock isolation}

Let us return to the problem defined by Eq. (15). In the problem, $u(t)$ is a function of time, which form is unknown. The simplest yet universal representation of an unknown function is a step function. Thus, we discretize $u(t)$ using the following step functions like Eq. (17):

$$
u=\left\{\begin{array}{l}
u_{1}, 0 \leqslant t \leqslant t_{1} \\
u_{2}, t_{1} \leqslant t \leqslant t_{2} \\
\ldots \ldots \quad \ldots \\
u_{n}, t_{n-1} \leqslant t \leqslant t_{n}
\end{array}\right.
$$

where $0<t_{1}<t_{1}<\ldots<t_{n}=t_{f}$ is a division of time and $u_{i}$ is constant in $\left[t_{i-1}, t_{i}\right]$. We may also write the above equation in the form $u=\left(u_{1}, u_{2}, \ldots, u_{n}\right)^{T}$. Then our problem is formulated as follows:

$$
\begin{aligned}
& \text { Find } u=\left(u_{1}, u_{2}, \ldots, u_{n}\right)^{T} \text { such that } \\
& \max |x(t)| \rightarrow \min \\
& m_{H} A \leqslant u(t) \leqslant m_{H} A,
\end{aligned}
$$




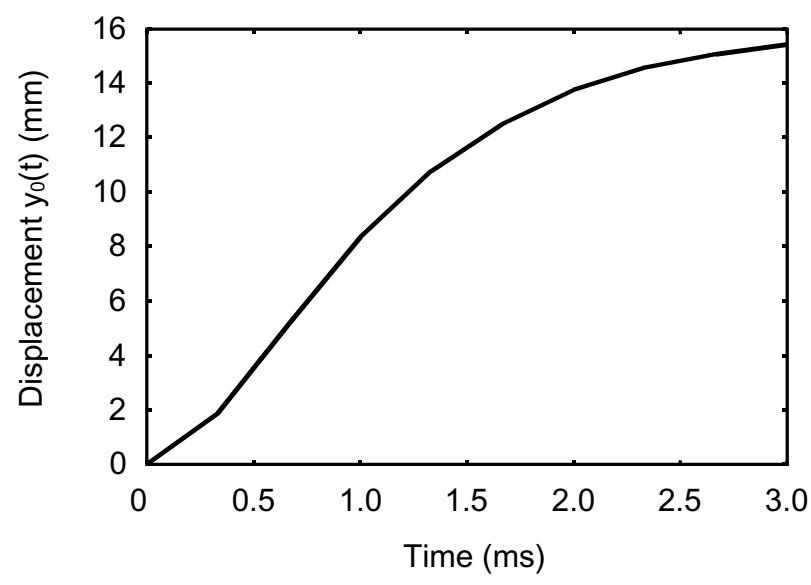

Fig. 7. Plate displacement in the absence of isolator and human body.

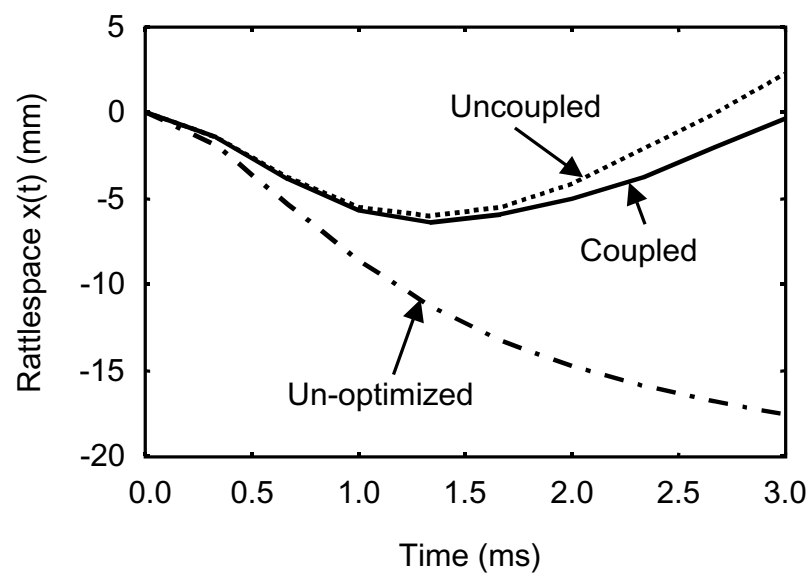

Fig. 8. Time history of optimized rattlespace.

where,

$$
\begin{aligned}
& x(t)=z(t)-y_{0}(t)-Y(t), \\
& m_{H} \ddot{z}=-u(t), \\
& m_{P} \ddot{Y}=u(t) .
\end{aligned}
$$

\section{Results and discussions}

Consider the following example problem: the charge weight $G=250 \mathrm{~kg}$, the standoff distance $R=10 \mathrm{~m}$, the human body mass $m_{H}=70 \mathrm{~kg}$, the plate mass $m_{P} S=1000 \mathrm{~kg}$, the acceleration constraint $A=$ $40 m_{H} g$ where $g$ is gravity acceleration. The force function is composed of $n=10$ step functions. Using GA, we optimized above problem with results shown in Figs 6-11.
Figure 6 shows the time histories of the plate acceleration with and without the human body taken into account. In this case where the plate mass is much greater than the human body's mass, the influence of human body is small. It can be seen that the acceleration is very large, much higher than the limit of human body tolerance. Figure 7 shows the displacement of the plate.

Figure 8 shows the time history of the optimized rattlespace. The "uncoupled" in the figure denotes the time history of the optimum rattlespace when the term in Eq. (5) is neglected (with isolator-structure interaction neglected). The "coupled" in the figure denotes the time history of the optimum rattlespace when the term $Y(t)$ in Eq. (5) is considered (structureisolation-human interaction). The "un-optimized" denotes the time history of the rattlespace for the given force: $u=\left(40 m_{H} g, 40 m_{H} g, \ldots, 40 m_{H} g\right)^{T}$. The 


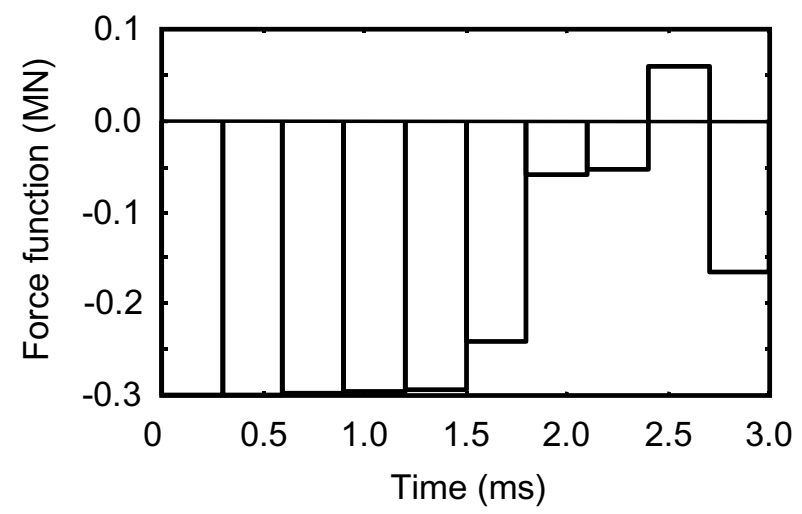

Fig. 9. Optimum force function.

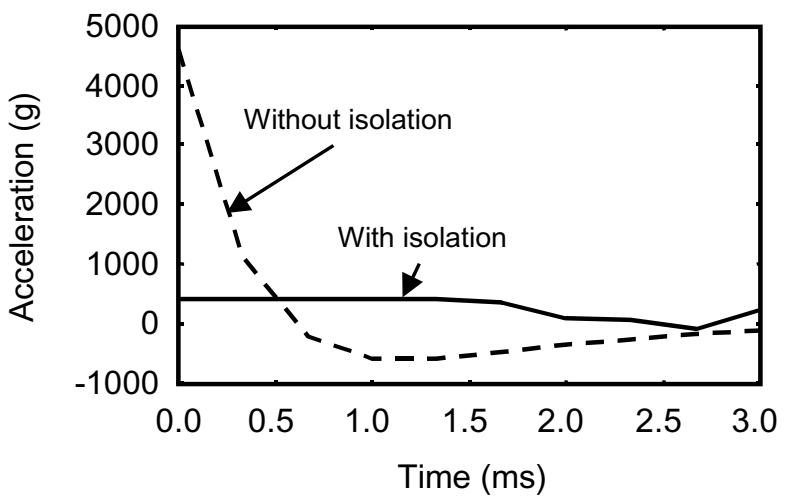

Fig. 10. Time histories of accelerations of the human body with and without isolation.

differences between "coupled" and "uncoupled" are not significant, both achieving the maximum displacement $6 \mathrm{~mm}$ at $t=1.5 \mathrm{~ms}$. $6 \mathrm{~mm}$ is the lower bound for the rattlespace whatever isolators are used. It is the theoretical limit of isolation effect. The corresponding optimized force function $u(t)=\left(u_{1}, u_{2}, \ldots, u_{10}\right)^{T}$ is shown in Fig. 9. The isolation effect of a specified isolator largely depends on the closeness of its force function to the function given in Fig. 9. Note the unit of the ordinate in Fig. 9 is MN, which is $10^{6}$ Newton.

In Fig. 10 are compared the isolation effects using the acceleration histories of the human body with and without isolation. The body acceleration with isolation is calculated using Eq. (1) and the data in Fig. 9. The acceleration of the human body is significantly reduced.

Figure 11 shows the searching process for the objective function (performance index) $\max |x(t)|$ and two components $u_{4}$ and $u_{6}$ in Eq. (19). After generation 100 , both the performance index and the variables $u_{4}$ and $u_{6}$ do not change too much. Their values at generation 300 are taken as the optimal values.
Note that all the above results, like the optimized rattlespace $X=\min \max |x(t)|$, are obtained under the constraint $A=40 m_{H} g$. A different acceleration constraint $A$ leads to another rattlespace optimum $X$. Thus, there exists a relationship between $X$ and $A$ known as limiting performance characteristic, and it can be obtained by repeatedly using the method above. Two such relations are shown in Fig. 12 for $R=10 \mathrm{~m}$ and $R=20 \mathrm{~m}$, respectively. The charge weight is same in these two cases with $G=250 \mathrm{~kg}$. In the figure, both $X$ and $A$ are normalized using normalizing constants $X_{m}$ and $A_{m}$. Their values are given in the figure. Figure 12 represents such a restriction that any design point below the curve, say $P_{1}$ in the figure, is a physically impossible isolator. Any design point on the curve, say $P_{2}$, is the optimum isolator that can physically be manufactured. Any design point above the curve, say $P_{3}$, represents a design that can be further improved. The margin for improvement is given by the distance between $P_{2}$ and $P_{3}$ [11]. Therefore, this figure provides the guidelines for isolator selection. 


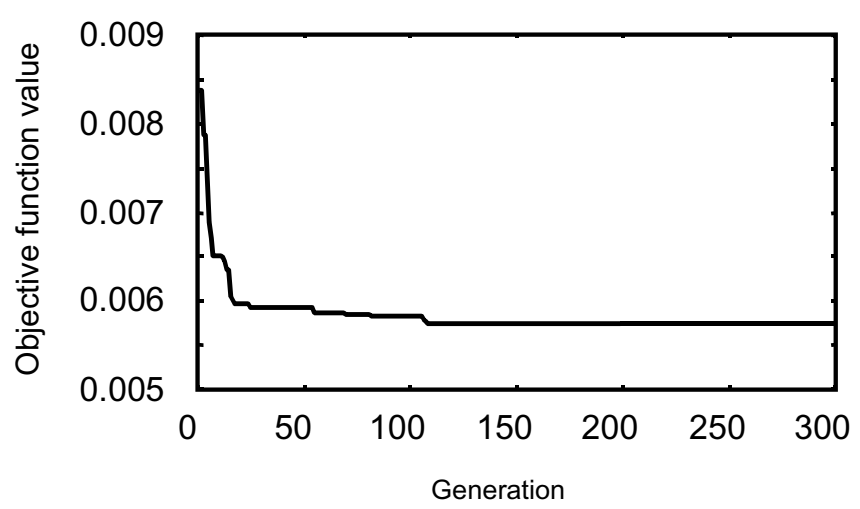

(a) Objective function values vs. generation

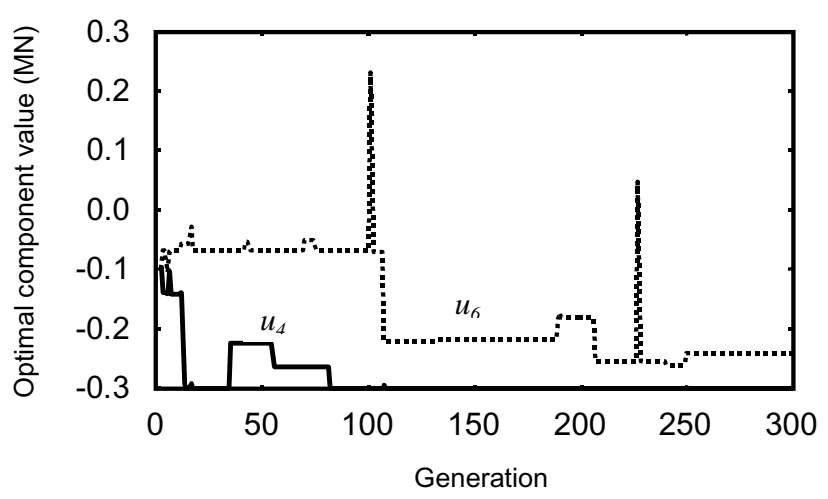

(b) Variable values vs. generation

Fig. 11. The search process for the optimum.

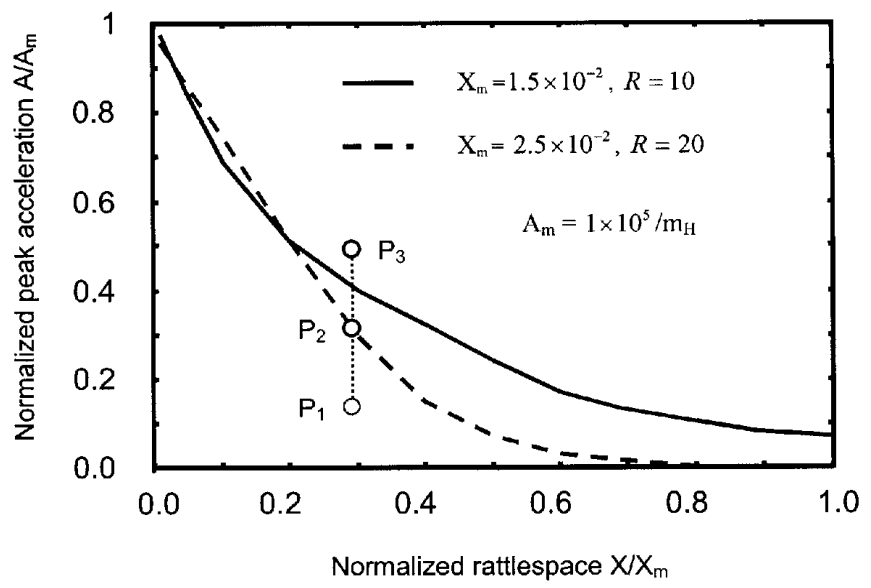

Fig. 12. Limiting performance characteristic.

\section{Conclusions}

In this paper, a SDF shock-structure-isolator-human interaction model was first given. The model incor- porates the effect of fluid-structure interaction, biodynamic response of human body and isolator influence. Based on this model, the optimum underwater shock isolation was formulated. The performance in- 
dex and restriction were defined. Using GA (genetic algorithm), we successfully solved the formulated optimization problem. Finally, the method presented in this paper was applied to an example, and the limiting performance characteristic was obtained.

\section{Acknowledgements}

The authors are grateful to David L. Carroll, University of Illinois at Urbana-Champaign, Urbana, Illinois 61801, for his GA driver and helpful suggestions.

\section{References}

[1] D.V. Balandin, N.N. Bolotnik and W.D. Pilkey, Optimal Protection from Impact, Shock, and Vibration, Gordon and Breach, NJ, 1999.

[2] D.V. Balandin, N.N. Bolotnik and W.D. Pilkey, On the Optimal Shock Isolation of a System with One and a Half Degrees of Freedom, Shock and Vibration 6 (1999), 159-167.

[3] R.H. Cole, Underwater Explosions, Princeton University Press, Princeton, NJ, 1948.

[4] J.A. DeRuntz Jr., The Underwater Shock Analysis Code and Its Applications, Proceedings, 60th Shock and Vibration Symposium, (vol. 1), David Taylor Research Centre, Bethesda, 1989, pp. 89-107.

[5] A.M. Eiband, Human Tolerance to Rapidly Applied Acceleration. A Survey of the Literature. National Aeronautics and Space Administration, Washington DC, NASA Memo No. 519-59E, 1959.
[6] M. Gen and R.W. Cheng, Genetic Algorithms and Engineering Design, A Wiley-Interscience Publication, John Wiley \& Sons, Inc., New York, 1997.

[7] K.Y. Lam, Z. Zong, G.W. Gong, T. Gan and Y.S. Shin, Human Exposure to Underwater Shock Modeling and Simulation, Naval Technology Seminar Platform, Singapore, Paper No. 4, 1999, pp. 1-15.

[8] X.X. Liu et al., Biodynamic Response and Injury Estimation of Ship Personnel to Ship Shock Motion Induced by Underwater Explosion, 69th Shock and Vibration Symposium, St. Paul, MN., 1998.

[9] MSC/DYTRAN, User's Manual, The Macheal-Schwendler Corporation, version 3.0, 1996.

[10] D.B. Oglesby and Y.S. Shin, Simulations of Human Male and Female Biodynamic Response to UNDEX Events, 69th Shock and Vibration Symposium, St. Paul, MN., 1998.

[11] S. Sevin and W.D. Pilkey, Optimum Shock and Vibration Isolation, The Shock and Vibration Information Center, United States Department of Defense, Washington, DC, 1971.

[12] Y.S. Shin and J.E. Chisum, Modeling and Simulation of Underwater Shock Problems Using a Coupled Lagrangian-Eulerian Analysis Approach, Shock and Vibration 4 (1997), 1-10.

[13] D.C. Viano and A.I. King, Injury Mechanisms and Biofidelity of Dummies, in: Crashworthiness of Transportation Systems: Structural Impact and Occupant Protection, J.A.C. Ambrosio et al., ed., Kluwer Academic Publishers, Dordrecht, 1997, pp. 25-51.

[14] Z. Zong, K.Y. Lam and G.R. Liu, Numerical Simulations of Scattering of an Underwater Shock Wave by a Half Cylinder Fixed on the Seabed, Proceedings, 3rd High Performance Computing Asia, (vol. 3), Singapore, 1998, pp. 983-988.

[15] Z. Zong, K.Y. Lam and G.R. Liu, Numerical Investigations of Damaging Effects of Underwater Shock on Submarine Structures in the Presence of Free Surface, 69th Shock and Vibration Symposium, St. Paul, MN., 1998. 

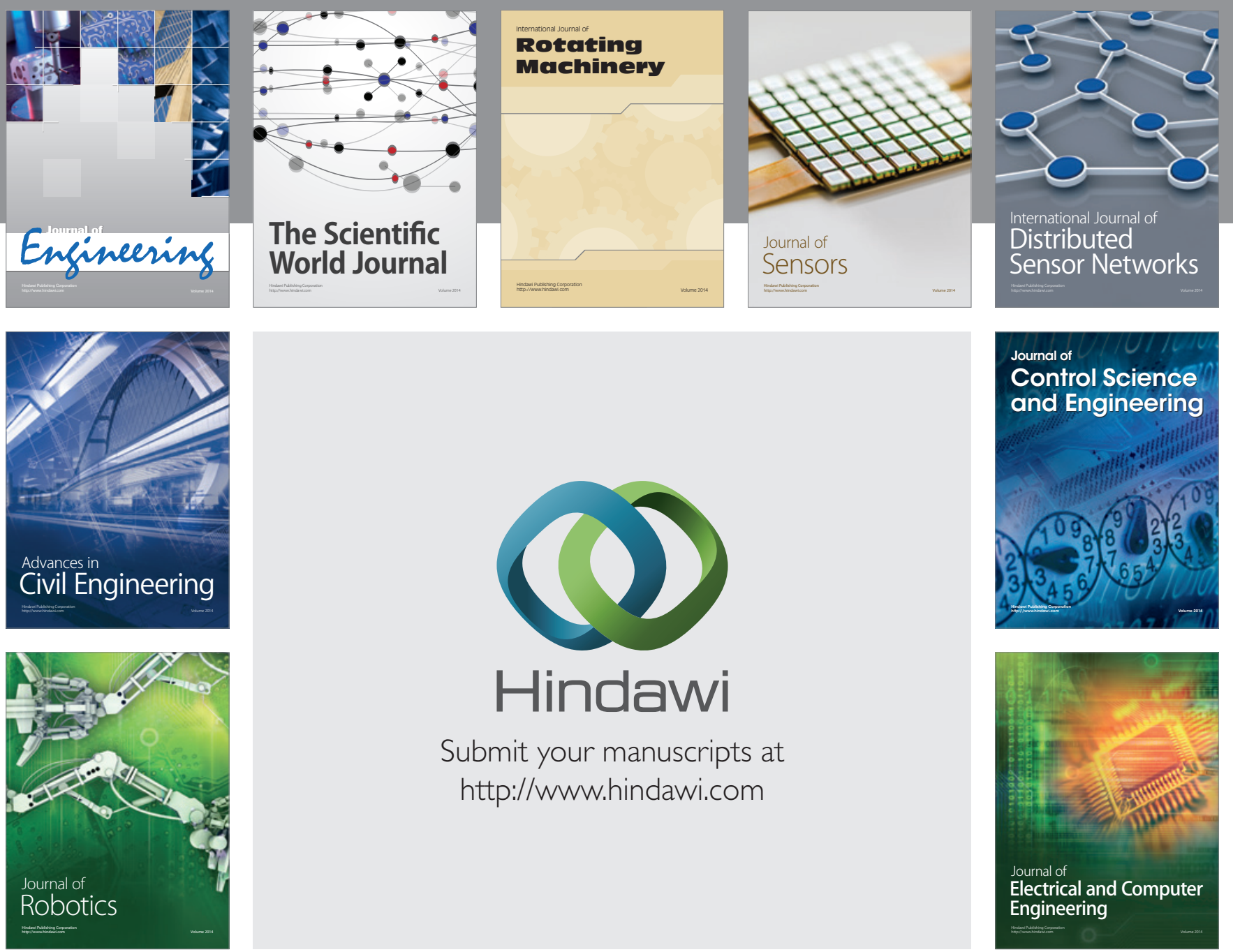

Submit your manuscripts at

http://www.hindawi.com
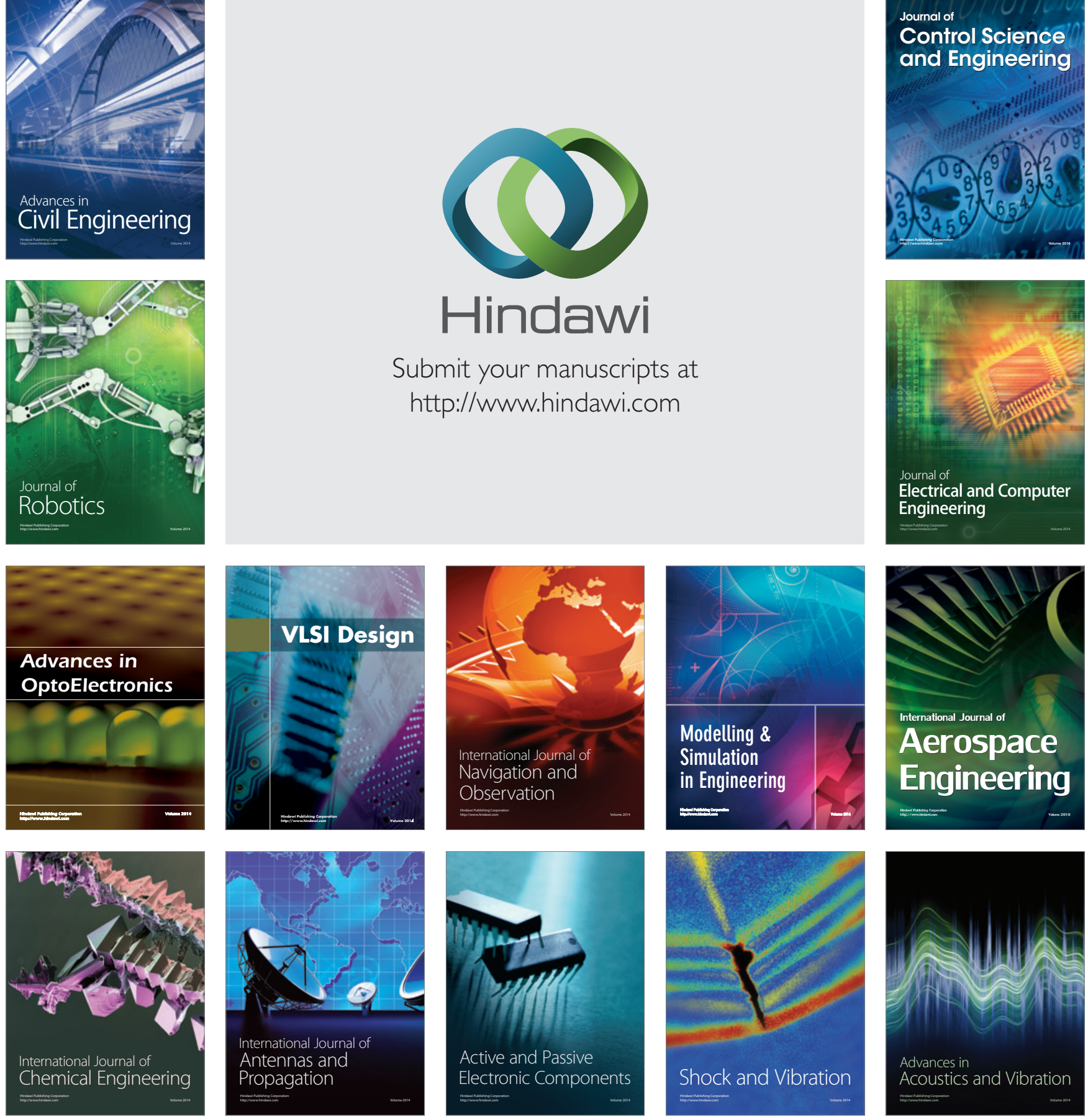